\title{
Kernos
}

Revue internationale et pluridisciplinaire de religion grecque antique

$10 \mid 1997$

Varia

\section{E. LIPPOLIS, S. GARRAFFO, M. NAFISSI, Taranto}

\section{Véronique Krings}

\section{(2) OpenEdition}

\section{Journals}

Édition électronique

URL : http://journals.openedition.org/kernos/694

DOI : $10.4000 /$ kernos. 694

ISSN : 2034-7871

Éditeur

Centre international d'étude de la religion grecque antique

Édition imprimée

Date de publication : 1 janvier 1997

Pagination : 363-364

ISSN : 0776-3824

Référence électronique

Véronique Krings, «E. LIPpolis, S. GarRafFo, M. nafISSI, Taranto », Kernos [En ligne], 10 | 1997, mis en ligne le 12 avril 2011, consulté le 21 septembre 2020. URL : http://journals.openedition.org/kernos/694 ;

DOI : https://doi.org/10.4000/kernos.694 
L'objectif principal que l'A. s'était fixé, en raison du public visé et du cadre restreint du livre, de faire avant tout prendre conscience des problèmes de connaissance qui se posent à l'historien de l'Occident archaïque est largement atteint. L'utilisateur de l'ouvrage se trouve en effet confronté à des documents de nature diverse, qui sont bien intégrés à la trame de l'exposé, ainsi qu'à une bibliographie moderne généralement bien amenée. L'A. a par ailleurs tenu à caractériser les Grecs d'Occident dans l'intention, plusieurs fois affirmée, non pas de les marginaliser mais bien de les insérer dans l'ensemble du monde grec méditerranéen. On notera toutefois que la Sicile et la Grande-Grèce ont davantage bénéficié de cet éclairage et que certains domaines, la religion ( $c f$. cependant la bibliographie p. 335-337) en particulier, n'ont guère retenu l'attention. Cet effort me semble en outre s'être parfois exercé à l'encontre des partenaires des Grecs en Méditerranée occidentale qui n'apparaissent ici qu'au détour, soit ponctuellement (p. ex., p. 179) soit de façon généralisante (p. ex., p. 87). Par ailleurs, les nombreuses illustrations, toujours bienvenues, sont citées sans référence à la source dont elles proviennent, ce qui est frustrant d'un point de vue scientifique et pédagogique. On signalera enfin un certain nombre de coquilles typographiques (p. ex., ipayges, p. 9; à utilisé p.12; Harpale, p. 55; éléveurs, p. 63; Pithécusses, p. 68; Inquiets ..., Sybaris, p. 79; moment ou ... Péniciens, p. 87; désormais, p. 114, etc), de manquement à l'uniformisation (tantôt Pseudo-Scymnos, tantôt Pseudo-Skymnos), de petites erreurs de détail (p. ex., ce n'est pas Strabon mais bien Diodore qui rapporte les détails de l'installation des rescapés de l'expédition de Pentathlos à Lipari, p. 84) ou de mise en page (p. ex., p. 160 en bas - 161 au dessus). Un index aurait été bien utile.

Véronique KRINGS

(Université de Liège)

Enzo Lippolis, Salvatore Garraffo \& Massimo Nafissi, Taranto, Taranto, Istituto per la storia e l'archeologia della Magna Grecia, 1995. 1 vol. $21 \times 29,5 \mathrm{~cm}$, 374 p., 64 pl. (Culti greci in Occidente. Fonti scritte e documentazione archeologica, I).

Le premier volume de la collection nouvellement créée «Culti greci in Occidente » est consacré à Tarente. D'autres, déjà annoncés en préparation, concerneront pour commencer les cités grecques de Grande-Grèce. Le sous-titre de la collection « Fonti scritte e documentazione archeologica » rend bien compte des intentions des A. Il s'agit de rassembler les testimonia afin de proposer un ensemble documentaire solide et à jour à l'intention de ceux qui s'intéressent à la religion des Grecs d'Occident. Les critères qui ont présidé au choix et à l'organisation de la documentation sont brièvement exposés (p. 11-13). Le corpus proprement dit est également précédé d'une introduction générale à l'histoire de Tarente (M. NAFISSI, p. 17-27). L'ensemble de la documentation est alors divisé en trois parties, l'exposé du matériel étant chaque fois précédé d'une présentation de nature plutôt méthodologique. La première partie (E. Lippolis, p. 29-129) est réservée à la documentation archéologique. Celle-ci est répartie en quatre grandes catégories sur la base du contexte topographique, à l'intérieur desquelles on retrouve les différentes classes de documents. La deuxième porte sur la documentation numismatique (S. GARRAFFo, p. 131-151) qui est organisée selon l'ordre alphabétique des divinités représentées. La troisième concerne la documentation littéraire et épigraphique (M. NAFISSI, p. 153-334) qui est répartie en sept grandes 
sections (le culte : divinités; le culte : héros; le culte : autres témoignages; le patrimoine mythico-religieux; la ktisis; la religion de la pólis en dehors de son territoire; les témoignages à caractère littéraire). En général, chaque inscription est accompagnée d'un lemme descriptif. Pour les textes littéraires, le contexte est parfois précisé, le texte grec ou latin est fourni, avec un apparat critique ne signalant que les variantes en rapport avec les noms des divinités ou des héros; une traduction est proposée, et, suivant les cas, un commentaire plus ou moins détaillé sur les principaux problèmes posés par le texte; on trouve enfin une bibliographie. L'intérêt de ce genre d'entreprise est patent, l'absence de recueils de sources rendant parfois fort difficile, voire impossible, le travail sur des dossiers où le matériel inédit ou d'accès difficile, en raison notamment du caractère local des publications, est abondant. De même, on apprécie grandement le souci des A. d'accompagner les documents d'un commentaire, lorsque cela leur paraît utile, dans l'intention de procéder à une première exégèse de ceux-ci. Il convient toutefois de ne pas perdre de vue qu'il s'agit alors déjà d'une interprétation historique qui, comme telle, portera nécessairement le poids des idées, des a priori de ceux qui les écrivent. L'ensemble est suivi d'une bibliographie, de différents index, d'un renvoi aux principales éditions de textes et, pour terminer, des planches photographiques.

Véronique KRINGS (Université de Liège)

Jutta Fischer, Griechisch-römische Terrakotten aus Ägypten. Die
Sammlungen Sieglin und Schreiber. Dresden, Leipzig, Stuttgart, Tübingen, Tübingen, Wasmuth, 1994. 1 vol. in $-4^{\circ}, 470$ p., 131 pl. (Tübinger Studien zur Archäologie und Kunstgeschichte, 14). Prix : 372 D.M.

La présente étude est consacrée aux très riches collections de terres cuites grécoromaines d'Égypte, rassemblées à la fin du siècle dernier et au début de ce siècle, par E. vON ZiEglin et Th. SCHREIBER, au départ de fouilles effectuées à Alexandric ou d'achats opérés dans la même ville, et dont une partie avait été publiée en 1924 par J. VOGT. Cet important corpus, riche de 1269 pièces, dispersées dans plusieurs villes d'Allemagne, est aujourd'hui l'objet d'une publication soignée, et magnifiquement illustrée, qui constitue une contribution essentielle à l'étude des productions sorties des ateliers des coroplathes depuis l'époque pré-ptolémaïque jusqu'au cœur de l'Égypte romaine. Ces terres cuites bénéficient depuis quelques années d'un regain d'intérêt, comme le prouve la publication de catalogues récents, dont G. NACHTERGAEL a rendu compte dans une chronique fondamentale (Terres cuites de l'Égypte grécoromaine. Á propos de quatre Catalogues récents, in CdE, 70 [1995], p. 254-294) pour l'étude et la compréhension de ces objets. Un dernier ouvrage consacré par L. TöröK aux terres cuites de Budapest, et que nous présentons dans le même numéro de cette revue, nous donne à espérer que ce mouvement ne s'arrêtera pas en si bon chemin.

L'ouvrage de Jutta FISCHER ne nous offre pas seulement un catalogue soigné de 357 pages, mais il commence par nous fournir une longue étude introductive (p. 1-105) mettant l'accent sur le développement typologique et chronologique des terres cuites.

Après avoir rappelé l'histoire de la collection (ch. I), l'A. se penche dans un ch. II sur les problèmes techniques (terre, technique et ateliers) qui permettent de distinguer trois types : les œuvres pré-ptolémaïques sorties des ateliers de Memphis, les figurines de haute époque hellénistique et les terres cuites dites du Fayoum, surtout d'époque 